\title{
Testable constraint on near-tribimaximal neutrino mixing
}

\author{
Biswajoy Brahmachari $^{a}$ and Probir Roy ${ }^{b, c}$ \\ ${ }^{a}$ Department of Physics, Vidyasagar Evening College, \\ 39 Sankar Ghosh Lane, Kolkata, 700006 India \\ ${ }^{b}$ Saha Institute of Nuclear Physics, \\ Kolkata, 700064 India \\ ${ }^{c}$ Center for Astroparticle Physics and Space Science, Bose Institute, \\ Kolkata, 700091 India \\ E-mail: biswa.brahmac@gmail.com, probirrana@gmail.com
}

ABSTRACT: General lowest order perturbations to hermitian squared mass matrices of leptons are considered away from the tribimaximal (TBM) limit in which a weak flavor basis with mass diagonal charged leptons is chosen. The three measurable TBM deviants are expressed linearly in terms of perturbation induced dimensionless coefficients appearing in the charged lepton and neutrino flavor eigenstates. With unnatural cancellations assumed to be absent and the charged lepton perturbation contributions to their flavor eigenstates argued to be small, we analytically derive the following result. Within lowest order perturbations, a deviation from maximal atmospheric neutrino mixing and the amount of $\mathrm{CP}$ violation in neutrino oscillations cannot both be large (i.e. 12-17\%), posing the challenge of verification to forthcoming experiments at the intensity frontier.

Keywords: Beyond Standard Model, Neutrino Physics, CP violation

ARXIV EPRINT: 1407.5293 


\section{Contents}

1 Introduction 1

2 Broken tribimaximal mixing and its effects 2

3 Lowest order perturbation away from tribimaximality 4

$\begin{array}{lll}4 & \text { Consequences of lowest order perturbation } & 7\end{array}$

$\begin{array}{lll}5 & \text { Results and discussion } & 9\end{array}$

6 Comparative studies with specific flavor models of broken TBM 10

$\begin{array}{lll}7 & \text { Concluding summary } & 12\end{array}$

$\begin{array}{ll}\text { A Derivation of mixing constraints } & 13\end{array}$

\section{Introduction}

The phenomenon of mixing between different generations of quarks/leptons has now been experimentally studied fairly well [1]. The three quark mixing angles are at present quite well-measured. Though the leptonic mixing angles are not known as well, one has credible nonzero $3 \sigma$ upper and lower bounds on them. $\mathrm{CP}$ violation has been investigated quite thoroughly in the quark sector, but as yet there is no reliable observation of $\mathrm{CP}$ violation involving only leptons. Quark mixing angles are known to become progressively smaller in order of magnitude as one moves from $1-2$ to $2-3$ and 1-3 generation mixing. This fact can be understood qualitatively in terms of a hierarchical quark mass matrix. The mixing angles, that emerge from such a mass matrix, are small and turn out to be given roughly by the mass ratios of relevant generations of quarks. Since the masses of both up- and down-type quarks are strongly hierarchical with respect to generations, this ties in with observation. In complete contrast, the leptonic mixing angles have been found to be much larger and show a different pattern. The qualitative difference between quark and lepton mixing patterns is made starkly evident by a quantitative comparison of the approximate magnitudes [2-5] of the elements of the respective unitary matrices $V_{C K M}$ and $U_{P M N S}$ :

$$
\left|V_{C K M}\right| \sim\left(\begin{array}{ccc}
0.9 & 0.2 & 0.004 \\
0.2 & 0.9 & 0.01 \\
0.008 & 0.04 & 0.9
\end{array}\right), \quad\left|U_{P M N S}\right| \sim\left(\begin{array}{ccc}
0.8 & 0.5 & 0.2 \\
0.4 & 0.6 & 0.7 \\
0.4 & 0.6 & 0.7
\end{array}\right) .
$$

Though the masses of the charged leptons $l(=e, \mu, \tau)$ show a pronounced hierarchical pattern with respect to generations, one suspects that such may not be the case with 
neutrinos. What operates for the mixing the latter, possibly related to their presumed Majorana nature ${ }^{1}$ originating, say from some kind of a seesaw mechanism [2], is perhaps some underlying family symmetry. Though one need not make any specific assumption on the neutrino mass hierarchy, such considerations are most natural for quasi-degenerate neutrinos. Even if there is any mass hierarchy among neutrinos, it can be presumed to be quite mild. Thus we separate the issue of the mixing of neutrinos from that of their mass hierarchy. More definitely, the family symmetry controlling their mixing can be taken to be independent of the neutrino mass hierarchy.

For fermions of type $t(=u, d, l, \nu)$, we can define the mass basis as one in which the corresponding mass matrix $M_{t}$ is diagonal. We can also consider the flavor basis in which the fermions $\left|\chi^{t}\right\rangle$ are flavor eigenstates but the mass matrix $M_{t f}$ is not necessarily diagonal. The hermitian squared mass matrix $M^{\dagger} M$ in each basis is related by a unitary transformation $U_{t}$ :

$$
U_{t}^{\dagger} M_{t f}^{\dagger} M_{t f} U_{t}=M_{t}^{\dagger} M_{t}
$$

We subscribe to the following viewpoint. While each of $U_{u}, U_{d}, U_{\ell}$ shows a hierarchical structure, this is not true of $U_{\nu}$ which is governed by a different principle. The way to gain new insights into this principle is through more precise measurements of the leptonic mixing angles and of the associated CP violating Dirac phase $\delta_{\mathrm{CP}}$ as well as of the concerned neutrino masses. These can test mixing constraints from specific theoretical ideas. Our aim in this paper is to derive some such constraint which is experimentally testable. This we do by considering lowest order perturbation theory in the additive breaking of tribimaximal (TBM) neutrino mixing for neutrino and charged lepton mass matrices in the flavor basis. The additively broken TBM paradigm is explained in detail below. From our consideration, we obtain two alternative experimentally testable possibilities, at least one of which is obligatory. Though our result is derived by use of general arguments, we check it in specific flavor models.

The rest of the paper is organized as follows. Section 2 is devoted to a discussion of tribimaximal mixing and its breaking. In section 3 we set up our basic lowest order perturbative formalism which is meant to compute the deviations away from tribimaximality. Section 4 contains the derivation of the theoretical consequences of the said formalism. In section 5 we discuss the experimentally testable constraint arising therefrom. Section 6 includes a comparative study of our result with those of various flavor models incorporating deviations from TBM. The final section 7 summarizes our conclusions.

\section{Broken tribimaximal mixing and its effects}

There is a vast literature [2-6] covering theoretical ideas on the principle governing $M_{\nu f}$ and $U_{\nu}$. Our focus, however, is on tribimaximal (TBM) mixing [6-9] which is elegant, predictive

\footnotetext{
${ }^{1}$ We follow the procedure of ref. [2] and take neutrinos to be light Majorana particles occurring in three generations. Consequently, we take a complex symmetric mass matrix for them. In the mass basis, that is $M_{\nu}=$ diag. $\left(m_{\nu 1}, m_{\nu 2}, m_{\nu 3}\right)$ with $m_{\nu 1}=\left|m_{\nu 1}\right|, m_{\nu 2}=\left|m_{\nu 2}\right| e^{-i \alpha_{21}}, m_{\nu 3}=\left|m_{\nu 3}\right| e^{-i \alpha_{31}}$ and $\alpha_{21}, \alpha_{31}$ as Majorana phases. We also use $c_{i j} \equiv \cos \theta_{i j}$ and $s_{i j} \equiv \sin \theta_{i j}$ for the angle of mixing $\theta_{i j}$ between neutrino flavors $i$ and $j$.
} 
and can be given a solid theoretical foundation $[10,11]$ from specific realizations of discrete family symmetries such as $A_{4}, S_{3}$ and $\Delta_{27}$. Some of the latter have also suggested a few neutrino mixing sum-rules [12-18]. We henceforth use the superscript zero to denote the TBM limit. In this limit we choose to work in the weak flavor basis in which the charged leptons have a diagonal Dirac mass matrix

$$
M_{\ell}^{0}=\operatorname{diag} .\left(m_{e}^{0}, m_{\mu}^{0}, m_{\tau}^{0}\right)
$$

The TBM limit of the neutrino mass matrix $M_{\nu f}^{0}$ in the flavor basis is characterized by certain linear relations among elements of $M_{\nu f}^{0}$ :

$$
\begin{aligned}
\left(M_{\nu f}^{0}\right)_{12} & =-\left(M_{\nu f}^{0}\right)_{13} \\
\left(M_{\nu f}^{0}\right)_{22} & =\left(M_{\nu f}^{0}\right)_{33} \\
\left(M_{\nu f}^{0}\right)_{11}-\left(M_{\nu f}^{0}\right)_{13} & =\left(M_{\nu f}^{0}\right)_{22}-\left(M_{\nu f}^{0}\right)_{23}
\end{aligned}
$$

Given (2.2), (2.3) and (2.4), the most general form of $M_{\nu f}^{0}$ is

$$
M_{\nu f}^{0}=\left(\begin{array}{ccc}
X & Y & -Y \\
Y & X+Z & -Y+Z \\
-Y & -Y+Z & X+Z
\end{array}\right),
$$

where $X, Y, Z$ are unknown complex mass dimensional parameters. Now the TBM pattern obtains with the three emergent pairwise mixing angles, that appear [1] in $U_{P M N S}$, being fixed at $\theta_{12}^{0}=\sin ^{-1} \sqrt{\frac{1}{3}} \simeq 35.3^{\circ}, \theta_{23}^{0}=\sin ^{-1} \sqrt{\frac{1}{2}}=45^{\circ}, \theta_{13}^{0}=0$ independent of whether the neutrino mass ordering is normal or inverted.

We can compare the TBM-predicted values of the three mixing angles with their current $3 \sigma$ allowed ranges. Recent global fits yield [19-21] $31^{\circ} \leq \theta_{12} \leq 36^{\circ}, 36^{\circ} \leq \theta_{23} \leq 55^{\circ}$, $7.2^{\circ} \leq \theta_{13} \leq 10^{\circ}$. Thus while $\theta_{12}$ and $\theta_{23}$ are certainly compatible with TBM values within their measured ranges, $\theta_{13}$ a fortiori is not. Indeed, the measurement of a significantly nonzero value of $\theta_{13}$ has been a major experimental advance recently [22-25] with a tremendous theoretical impact. This is due to two reasons. First, CP-violation, that is observable in neutrino oscillations, enters through the terms $s_{13} e^{ \pm i \delta_{\mathrm{CP}}}$; thus $s_{13} \sim 0.12$ 0.17 is very encouraging to that end. Second, it means that any symmetry, leading to TBM, must be a broken symmetry. The next natural question is: how quantitative is this breaking and is TBM still relevant in an approximate sense?

We make an attempt to answer this last question. Our approach is to add small general perturbations to the TBM limits of hermitian squared mass matrices $M_{\ell f}^{\dagger} M_{\ell f}$ and $M_{\nu f}^{\dagger} M_{\nu f}$. We take both sets of perturbations to be of the same order of magnitude and treat them to the lowest order. Much effort [26-29] has already been expended in this direction. However, we do have something new and interesting to say. We bring out a novel feature of the nearTBM mixing of neutrinos in terms of an analytically derived constraint which merits being highlighted. The constraint implies that at least one of two conditions, that are testable in forthcoming neutrino oscillation experiments, must hold. Either the deviation $\left|s_{23}-\sqrt{\frac{1}{2}}\right|$ from the maximal value of $\theta_{23}$ or the measure of $\mathrm{CP}$ violation $\left|s_{13} \sin \delta_{\mathrm{CP}}\right|$ has to be quite 
small ( $<3 \%$ as opposed to ${ }^{2} 12-17 \%$ for the value of $s_{13}$ as compared with unity), the latter meaning that $\mathrm{CP}$ will be conserved at the lowest order. This conclusion is a consequence of the fact that the perturbed eigenstates $\left|\chi_{i}^{\ell, \nu}\right\rangle$ for $i=1,2,3$ make up the columns of the matrices $U_{\ell, \nu}$ to the lowest order. Hence any observation in the near future of both a sizable deviation from maximal atmospheric neutrino mixing and a large amount of $\mathrm{CP}$ violation in neutrino oscillations would go against the idea of lowest order additive perturbation to TBM-invariant neutrino and charged lepton mass matrices.

In deriving the above conclusion, we do not assume any additional model either at a high or at a low scale, or any specific discrete family symmetry. In fact, we perform a lowest order model independent analysis with the most general TBM violating perturbation matrices whose nonzero elements are expected to be of the same order of magnitude. Moreover, our results on neutrino mixing do not need to assume anything about the neutrino mass ordering. This is since the perturbations are expected to be some kind of symmetry breaking terms, which characterize their contributions to $\left|\chi^{\ell, \nu}\right\rangle$ by a set of small dimensionless coefficients $\left\{\epsilon^{\ell, \nu}\right\}$. All members of the subset $\left\{\epsilon^{\nu}\right\}$ in the neutrino sector are taken to be typically of magnitude $\sim s_{13} \equiv \sin \theta_{13} \sim 0.12-0.17$, i.e. of the order of $12-17 \%$ or thereabouts of the unperturbed quantities. On the other hand, in the charged lepton sector, arguements are given why $\left\{\epsilon^{\ell}\right\}$ are much less in magnitude than $\left\{\epsilon^{\nu}\right\}$ on account of the strongly mass hierarchical nature of the charged leptons. This will be shown to follow from all nonzero perturbation matrix elements being taken to be of the same order of magnitude. Of course, the neglected $O\left(\epsilon^{2}\right)$ terms are estimated to be only at a $2-3 \%$ level which is below [30] the accuracy of the measurement of TBM deviants in ongoing and forthcoming neutrino oscillation experiments. ${ }^{3}$

\section{Lowest order perturbation away from tribimaximality}

For charged leptons $\ell$ the normalized eigenvectors in the mass basis and the flavor basis are identical in the TBM limit. Thus we can take

$$
\left|\chi_{1}^{\ell 0}\right\rangle=\left|\chi_{1}^{\ell 0}\right\rangle_{f}=\left(\begin{array}{l}
1 \\
0 \\
0
\end{array}\right), \quad\left|\chi_{2}^{\ell 0}\right\rangle=\left|\chi_{2}^{\ell 0}\right\rangle_{f}=\left(\begin{array}{l}
0 \\
1 \\
0
\end{array}\right), \quad\left|\chi_{3}^{\ell 0}\right\rangle=\left|\chi_{3}^{\ell 0}\right\rangle_{f}=\left(\begin{array}{l}
0 \\
0 \\
1
\end{array}\right) .
$$

Moreover, the charged lepton mass matrix is identical in each basis in the same limit, namely

$$
M_{\ell f}^{0}=M_{\ell}^{0} .
$$

\footnotetext{
${ }^{2}$ We shall throughout refer to a TBM deviating effect as (1) "very large" if it is $\gg 100 s_{13} \% \sim 12-17 \%$ so that higher order perturbations cannot be ignored, (2) "large" if it is in the ballpark of $100 s_{13} \%$ so that it should be soon measurable as well as computable with only lowest order perturbations and (3) negligibly "small" if it is $<0.03$ which is $O\left(s_{13}^{2}\right)$.

${ }^{3}$ Experiments in the far future with neutrino factories may probe such a level and, for such measurements, the neglected $O\left(\epsilon^{2}\right)$ effects as well as those due to renornalization group evolution from an assumed high scale symmetry would be relevant.
} 
Adding a perturbation $M_{\ell f}^{\prime}\left(\equiv \lambda_{i j}\right)$ to $M_{\ell f}^{0}$ so that $M_{\ell f}=M_{\ell f}^{0}+M_{\ell f}^{\prime}$, we can construct the corresponding matrix $M_{\ell}^{\prime}$ in the mass basis as

$$
M_{\ell}=M_{\ell}^{0}+M_{\ell}^{\prime}
$$

via,

$$
M_{\ell}^{\dagger} M_{\ell}=U_{\ell}^{\dagger} M_{\ell f}^{\dagger} M_{\ell f} U_{\ell}
$$

Turning to neutrinos in the TBM limit, we can write

$$
U_{\nu}^{0 \dagger} M_{\nu f}^{0}{ }^{\dagger} M_{\nu f}^{0} U_{\nu}^{0}=\text { diag. }\left(\left|m_{\nu 1}^{0}\right|^{2},\left|m_{\nu 2}^{0}\right|^{2},\left|m_{\nu 3}^{0}\right|^{2}\right)
$$

with

$$
U_{\nu}^{0}=\left(\begin{array}{ccc}
\sqrt{2 / 3} & \sqrt{1 / 3} & 0 \\
-\sqrt{1 / 6} & \sqrt{1 / 3} & \sqrt{1 / 2} \\
\sqrt{1 / 6} & -\sqrt{1 / 3} & \sqrt{1 / 2}
\end{array}\right) .
$$

The normalized flavor eigenvectors $\left|\chi_{i}^{\nu 0}\right\rangle$ of $M_{\nu f}^{0 \dagger} M_{\nu f}^{0}$ for $i=1,2,3$ are the columns of $U_{\nu}^{0}$ while those in the mass basis are identical to the charged lepton ones. Thus

$$
\left|\chi_{1}^{\nu 0}\right\rangle=\left(\begin{array}{l}
1 \\
0 \\
0
\end{array}\right), \quad\left|\chi_{2}^{\nu 0}\right\rangle=\left(\begin{array}{l}
0 \\
1 \\
0
\end{array}\right), \quad\left|\chi_{3}^{\nu 0}\right\rangle=\left(\begin{array}{l}
0 \\
0 \\
1
\end{array}\right)
$$

whereas

$$
\left|\chi_{1}^{\nu 0}\right\rangle_{f}=\left(\begin{array}{c}
\sqrt{\frac{2}{3}} \\
-\sqrt{\frac{1}{6}} \\
\sqrt{\frac{1}{6}}
\end{array}\right), \quad\left|\chi_{2}^{\nu 0}\right\rangle_{f}=\left(\begin{array}{c}
\sqrt{\frac{1}{3}} \\
\sqrt{\frac{1}{3}} \\
-\sqrt{\frac{1}{3}}
\end{array}\right), \quad\left|\chi_{3}^{\nu 0}\right\rangle_{f}=\left(\begin{array}{c}
0 \\
\sqrt{\frac{1}{2}} \\
\sqrt{\frac{1}{2}}
\end{array}\right) .
$$

Once the perturbation is introduced, we have $M_{\nu f}=M_{\nu f}^{0}+M_{\nu f}^{\prime}$, where $M_{\nu f}^{0}$ obey the TBM conditions $(2.2)-(2.4)$ while $\left(M_{\nu f}^{\prime}\right)_{i j} \equiv \mu_{i j}=\mu_{j i}$ violate them. The violation in TBM conditions is given by,

$$
\begin{aligned}
\left(M_{\nu f}\right)_{12}+\left(M_{\nu f}\right)_{13} & =\mu_{12}+\mu_{13}, \\
\left(M_{\nu f}\right)_{22}-\left(M_{\nu f}\right)_{33} & =\mu_{22}-\mu_{33}, \\
\left(M_{\nu f}\right)_{11}-\left(M_{\nu f}\right)_{13}-\left(M_{\nu f}\right)_{22}+\left(M_{\nu f}\right)_{23} & =\mu_{11}-\mu_{13}-\mu_{22}+\mu_{23} .
\end{aligned}
$$

Note that, unlike the real diagonal $M_{\ell}^{0}$ and the general $M_{\ell}^{\prime}$, both $M_{\nu f}^{0}$ and $M_{\nu f}^{\prime}$ have to be complex symmetric matrices in order to make the corresponding neutrinos Majorana particles.

We now expand the perturbed eigenstates for both charged leptons and neutrinos at the lowest order. We choose to use a compact notation covering both cases by introducing perturbation parameters $\epsilon_{i k}^{\nu, \ell}$ (for $i, k=1,2,3$ ). Thus we can write the ith first order perturbed eigenvectors of $M_{\nu f}^{\dagger} M_{\nu f}$ on one hand and of $M_{\ell f}^{\dagger} M_{\ell f}$ on the other as

$$
\left|\chi_{i}^{\nu, \ell}\right\rangle_{f}=\left|\chi_{i}^{0 \nu, \ell}\right\rangle_{f}+\sum_{k \neq i} \epsilon_{i k}^{\nu, \ell}\left|\chi_{k}^{0 \nu, \ell}\right\rangle_{f}+O\left(\epsilon^{2}\right)
$$


Two new quantities have been introduced in (3.12). They are defined by

$$
\begin{aligned}
\epsilon_{i k}^{\nu, \ell} & =-\epsilon^{\nu, \ell^{*}}{ }_{k i}=\left(\left|m_{\nu, \ell i}^{0}\right|^{2}-\left|m_{\nu, \ell k}^{0}\right|^{2}\right)^{-1} p_{k i}^{\nu, \ell}, \\
p_{i k}^{\nu, \ell} & =\left\langle\chi_{i}^{0 \nu, \ell}\left|M_{\nu, \ell}^{0}{ }^{\dagger} M_{\nu, \ell}^{\prime}+M_{\nu, \ell}^{\prime} M_{\nu, \ell}^{0}\right| \chi_{k}^{0 \nu, \ell}\right\rangle .
\end{aligned}
$$

Note that (3.13) and (3.14) have been written in the mass basis utilizing the fact that $\epsilon_{i k}^{\nu, \ell}$, as well as $p_{i k}^{\nu, \ell}$, do not change from one basis to the other. We can also comment on the lack of dependence of the epsilon parameters on the yet unknown overall neutrino mass scale. If $M_{\nu}^{0}$ and $M_{\nu}^{\prime}$ are both scaled by a factor $\alpha$, the unperturbed eigenvalues $\left\{m_{\nu i}^{0}\right\}$ will also be scaled similarly. As a result, $\epsilon_{i k}^{\nu}$ will remain invariant under an overall mass scaling. On the other hand, suppose two of the mass eigenvalues are large but close to one another, as is the case with $\nu_{1}$ and $\nu_{2}$ with an inverted mass hierarchy, and this is not much affected by the perturbations. In such a case the corresponding $\epsilon_{12}^{\nu}$ will get enhanced.

Turning to (3.12), we see that its 1.h.s. for $i=1,2,3$ can be identified with three corresponding columns of $U_{\nu, \ell}$. Thus

$$
U_{\nu, l}=\left(\left|\chi_{1}^{\nu, \ell}\right\rangle_{f}\left|\chi_{2}^{\nu, \ell}\right\rangle_{f}\left|\chi_{3}^{\nu, \ell}\right\rangle_{f}\right)
$$

Neglecting $O\left(\epsilon^{2}\right)$ terms, it follows from (3.15) that

$$
U_{\ell}=\left(\begin{array}{ccc}
1 & -\epsilon_{12}^{\ell *} & -\epsilon_{13}^{\ell *} \\
\epsilon_{12}^{\ell} & 1 & -\epsilon_{23}^{\ell *} \\
\epsilon_{13}^{\ell} & \epsilon_{23}^{\ell} & 1
\end{array}\right)
$$

and

$$
U_{\nu}=\left(\begin{array}{ccc}
\sqrt{\frac{2}{3}}+\sqrt{\frac{1}{3}} \epsilon_{12}^{\nu} & \sqrt{\frac{1}{3}}-\sqrt{\frac{2}{3}} \epsilon_{12}^{\nu *} & -\sqrt{\frac{2}{3}} \epsilon_{13}^{\nu *}-\sqrt{\frac{1}{3}} \epsilon_{23}^{\nu *} \\
-\sqrt{\frac{1}{6}}+\sqrt{\frac{1}{3}} \epsilon_{12}^{\nu}+\sqrt{\frac{1}{2}} \epsilon_{13}^{\nu} & \sqrt{\frac{1}{3}}+\sqrt{\frac{1}{6}} \epsilon_{12}^{\nu *}+\sqrt{\frac{1}{2}} \epsilon_{23}^{\nu} & \sqrt{\frac{1}{2}}+\sqrt{\frac{1}{6}} \epsilon_{13}^{\nu *}-\sqrt{\frac{1}{3}} \epsilon_{23}^{\nu *} \\
\sqrt{\frac{1}{6}}-\sqrt{\frac{1}{3}} \epsilon_{12}^{\nu}+\sqrt{\frac{1}{2}} \epsilon_{13}^{\nu} & -\sqrt{\frac{1}{3}}-\sqrt{\frac{1}{6}} \epsilon_{12}^{\nu *}+\sqrt{\frac{1}{2}} \epsilon_{23}^{\nu} & \sqrt{\frac{1}{2}}-\sqrt{\frac{1}{6}} \epsilon_{13}^{\nu *}+\sqrt{\frac{1}{3}} \epsilon_{23}^{\nu *}
\end{array}\right) .
$$

Let us define the Majorana phase matrix

$$
K \equiv \operatorname{diag} .\left(1, e^{\frac{i \alpha_{21}}{2}}, e^{\frac{i \alpha_{31}}{2}}\right) .
$$

Then $U_{P M N S} K^{-1}$ can be written in the PDG convention [1] as

$$
U_{P M N S} K^{-1}=\left(\begin{array}{ccc}
c_{12} c_{13} & s_{12} c_{13} & s_{13} e^{-i \delta_{\mathrm{CP}}} \\
-s_{12} c_{23}-c_{12} s_{23} s_{13} e^{i \delta_{\mathrm{CP}}} & c_{12} c_{23}-s_{12} s_{23} s_{13} e^{i \delta_{\mathrm{CP}}} & s_{23} c_{13} \\
s_{12} s_{23}-c_{12} c_{23} s_{13} e^{i \delta_{\mathrm{CP}}} & -c_{12} s_{23}-s_{12} c_{23} s_{13} e^{i \delta_{\mathrm{CP}}} & c_{23} c_{13}
\end{array}\right) .
$$

We can now make the identification

$$
U_{P M N S} K^{-1}=U_{\ell}^{\dagger} U_{\nu}
$$

and work out the consequences from (3.16), (3.17) and (3.19). 


\section{Consequences of lowest order perturbation}

Let us define $L \equiv U_{\ell}^{\dagger} U_{\nu}$ and $N \equiv U_{P M N S} K^{-1}$. The identification $L_{i j}=N_{i j}$ as per (3.20) leads to nine equations which are detailed in convenient combinations in the appendix. Not all of these are independent, but they lead to four independent constraint conditions and three equations for the three TBM deviants $c_{12}-\sqrt{\frac{2}{3}}, c_{23}-s_{23}$ and $s_{13} e^{i \delta_{\mathrm{CP}}}$. The constraint conditions follow from the fact that four of the elements of $N$ are real. They are given by

$$
\begin{aligned}
\operatorname{Im} \epsilon_{12}^{\nu} & =O\left(\epsilon^{2}\right), \\
\operatorname{Im}\left(\epsilon_{13}^{\nu}-\sqrt{2} \epsilon_{23}^{\nu}\right) & =O\left(\epsilon^{2}\right), \\
\operatorname{Im} \epsilon_{23}^{l} & =O\left(\epsilon^{2}\right), \\
\operatorname{Im}\left(\epsilon_{12}^{l}-\epsilon_{13}^{l}\right) & =O\left(\epsilon^{2}\right) .
\end{aligned}
$$

Neglecting $O\left(\epsilon^{2}\right)$ terms, the three measurable TBM-deviants are linear in the $\epsilon$ coefficients and may be given as

$$
\begin{aligned}
c_{12}-\sqrt{2 / 3}=\sqrt{1 / 2}\left(\sqrt{1 / 3}-s_{12}\right) & =\sqrt{1 / 3} \epsilon_{12}^{\nu}-\sqrt{1 / 6}\left(\epsilon_{12}^{l}-\epsilon_{13}^{l}\right), \\
c_{23}-s_{23} & =-\sqrt{2 / 3}\left(\epsilon_{13}^{\nu}-\sqrt{2} \epsilon_{23}^{\nu}\right)-\sqrt{2} \epsilon_{23}^{l}, \\
s_{13} e^{i \delta_{\mathrm{CP}}} & =-\sqrt{1 / 3}\left(\sqrt{2} \epsilon_{13}^{\nu}+\epsilon_{23}^{\nu}\right)+\sqrt{1 / 2}\left(\epsilon_{12}^{l}+\epsilon_{13}^{l}\right) .
\end{aligned}
$$

The derivation of eqs. (4.1)-(4.7) appears in the appendix.

Because of (4.2), the real and imaginary parts of (4.7) enable us to write, modulo $O\left(\epsilon^{2}\right)$ terms, that

$$
\tan \delta_{\mathrm{CP}}=\frac{3 \operatorname{Im} \epsilon_{23}^{\nu}-\sqrt{3 / 2} \operatorname{Im}\left(\epsilon_{12}^{\ell}+\epsilon_{13}^{\ell}\right)}{\operatorname{Re}\left(\sqrt{2} \epsilon_{13}^{\nu}+\epsilon_{23}^{\nu}\right)-\sqrt{3 / 2} \operatorname{Re}\left(\epsilon_{12}^{l}+\epsilon_{13}^{l}\right)} .
$$

The above equation may be recast in terms of the basis independent Jarlskog invariant $J$ which equals

$$
\operatorname{Im}\left[\left(U_{\ell}^{\dagger} U_{\nu}\right)_{e 1}\left(U_{\ell}^{\dagger} U_{\nu}\right)_{\mu 2}\left(U_{\ell}^{\dagger} U_{\nu}\right)_{e 2}^{*}\left(U_{\ell}^{\dagger} U_{\nu}\right)_{\mu 1}^{*}\right]
$$

We then have

$$
J=-\frac{1}{\sqrt{6}} \operatorname{Im}\left[\epsilon_{23}^{\nu}-\frac{1}{\sqrt{6}}\left(\epsilon_{12}^{l}+\epsilon_{13}^{l}\right)\right]+O\left(\epsilon^{2}\right) .
$$

Let us now explore, to the lowest order in $\epsilon$, the consequences of (3.13) and (3.14) by explicitly taking elements of the respective perturbing mass matrices for neutrinos and charged leptons. We take

$$
\left(M_{\nu f}^{\prime}\right)_{i j}=\mu_{i j}=\mu_{j i}
$$

and

$$
\left(M_{\ell f}^{\prime}\right)_{i j}=\left(M_{\ell}^{\prime}\right)_{i j}+O\left(\epsilon^{2}\right)=\lambda_{i j}
$$

with $\lambda_{i j}$ and $\mu_{i j}=\mu_{j i}$ as complex mass dimensional parameters naturally expected to be of the same order of magnitude. The identity of the charged lepton mass basis and 
flavor basis in the TBM limit makes the calculations in this case quite straightforward. From (3.13) and (3.14), we can easily derive

$$
\begin{aligned}
\epsilon_{12}^{l} & =\left(m_{e}^{0^{2}}-m_{\mu}^{0^{2}}\right)^{-1}\left(m_{\mu}^{0} \lambda_{21}+m_{e}^{0} \lambda_{12}^{*}\right), \\
\epsilon_{23}^{l} & =\left(m_{\mu}^{0^{2}}-m_{\tau}^{0^{2}}\right)^{-1}\left(m_{\tau}^{0} \lambda_{32}+m_{\mu}^{0} \lambda_{23}^{*}\right), \\
\epsilon_{13}^{l} & =\left(m_{e}^{0^{2}}-m_{\tau}^{0^{2}}\right)^{-1}\left(m_{\tau}^{0} \lambda_{31}+m_{e}^{0} \lambda_{13}^{*}\right) .
\end{aligned}
$$

We want to comment on the magnitudes of $\epsilon_{23}^{\ell}$ and $\epsilon_{13}^{\ell}$. In order for them to be large, the relevant $\lambda$ parameters would need to be of order $m_{\tau}$. That is not in conformity with our premise that nonzero charged lepton perturbation mass matrix elements (i.e. $\lambda_{i j}$ ) cannot be very different in order of magnitude from those for neutrinos (i.e. $\mu_{i j}$ ). Thus we expect that $\left|\epsilon_{23}^{\ell}\right|$ and $\left|\epsilon_{13}^{\ell}\right|$ to be quite small. In any event, because of the strongly hierarchical nature of charged lepton masses, (4.3) and (4.4) can be satisfied without unnatural cancellations only by $\lambda_{12}, \lambda_{21}, \lambda_{13}, \lambda_{31}, \lambda_{23}, \lambda_{32}$ all being real to order $\epsilon$. One then automatically obtains that

$$
\operatorname{Im} \epsilon_{12}^{l}=O\left(\epsilon^{2}\right)=\operatorname{Im} \epsilon_{13}^{l} .
$$

Feeding this information, we can simplify (4.8) and (4.9) to

$$
\begin{aligned}
\tan \delta_{\mathrm{CP}} & =\frac{3 \operatorname{Im} \epsilon_{23}^{\nu}}{\operatorname{Re}\left(\sqrt{2} \epsilon_{13}^{\nu}+\epsilon_{23}^{\nu}\right)-\sqrt{3 / 2} \operatorname{Re}\left(\epsilon_{12}^{l}+\epsilon_{13}^{l}\right)}, \\
J & =-\frac{1}{\sqrt{6}} \operatorname{Im} \epsilon_{23}^{\nu}+O\left(\epsilon^{2}\right)
\end{aligned}
$$

respectively.

Turning to neutrinos next, the relevant off-diagonal elements of $M_{\nu}^{\prime}=U_{\nu}^{0^{T}} M_{\nu f}^{\prime} U_{\nu}^{0}$ are

$$
\begin{aligned}
& \left(M_{\nu}^{\prime}\right)_{12}=\frac{1}{3 \sqrt{2}}\left(2 \mu_{11}+\mu_{12}-\mu_{13}-\mu_{22}+2 \mu_{23}-\mu_{33}\right) \\
& \left(M_{\nu}^{\prime}\right)_{23}=\frac{1}{\sqrt{6}}\left(\mu_{12}+\mu_{13}+\mu_{22}-\mu_{33}\right) \\
& \left(M_{\nu}^{\prime}\right)_{13}=\frac{1}{\sqrt{3}}\left(\mu_{12}+\mu_{13}-\frac{1}{2} \mu_{22}+\frac{1}{2} \mu_{33}\right)
\end{aligned}
$$

It is now convenient to define

$$
\begin{aligned}
\Delta_{i j}^{0} & \equiv\left|m_{\nu i}^{0}\right|^{2}-\left|m_{\nu j}^{0}\right|^{2}, \\
a_{i j}^{\mp} & \equiv m_{\nu i}^{0} \mp m_{\nu j}^{0} .
\end{aligned}
$$

Then we take (3.13) and (3.14) and successively consider the index combinations $i=1, k=$ 2 and $i=2, k=3$ as well as $i=1, k=3$. Separating the real and imaginary parts and using (4.21) and (4.22), we obtain the following six equations

$$
2 \Delta_{12}^{0}\left(\begin{array}{c}
\mathrm{i} \operatorname{Im} \epsilon_{12}^{\nu} \\
\operatorname{Re} \epsilon_{12}^{\nu}
\end{array}\right)=a_{21}^{\mp_{2}^{*}}\left(M_{\nu}^{\prime}\right)_{12} \mp c . c .,
$$




$$
\begin{aligned}
& 2 \Delta_{23}^{0}\left(\begin{array}{c}
\mathrm{i} \operatorname{Im} \epsilon_{23}^{\nu} \\
\operatorname{Re} \epsilon_{23}^{\nu}
\end{array}\right)=a_{32}^{\mp^{*}}\left(M_{\nu}^{\prime}\right)_{23} \mp \text { c.c. }, \\
& 2 \Delta_{13}^{0}\left(\begin{array}{c}
\mathrm{i} \operatorname{Im} \epsilon_{13}^{\nu} \\
\operatorname{Re} \epsilon_{13}^{\nu}
\end{array}\right)=a_{31}^{\mp^{*}}\left(M_{\nu}^{\prime}\right)_{13} \mp \text { c.c. }
\end{aligned}
$$

Needless to add, order $\epsilon^{2}$ terms have been neglected in deriving the above results.

\section{$5 \quad$ Results and discussion}

Eq. (4.23) has a simple consequence if we exclude unnatural cancellations. In conjunction with (4.1), it forces the combination of $\mu_{i j}$, occurring in $\left(M_{\nu}^{\prime}\right)_{12}$, i.e. $2 \mu_{11}+\mu_{12}-\mu_{13}-\mu_{22}+$ $2 \mu_{23}-\mu_{33}$, to be real. It also implies that $m_{\nu_{2}}^{0}-m_{\nu_{1}}^{0}$ is real, the latter forcing $\alpha_{21}^{0}$ to be 0 or $\pi$. However, our key observation follows from combining (4.24) and (4.25) with (4.2). That procedure yields the equality

$\operatorname{Im}\left[\left(m_{\nu_{3}}^{0 *}-m_{\nu_{2}}^{0 *}\right)\left(\mu_{12}+\mu_{13}+\mu_{22}-\mu_{33}\right)\right]=\operatorname{Im}\left[\left(m_{\nu_{3}}^{0 *}-m_{\nu_{1}}^{0 *}\right)\left(\mu_{12}+\mu_{13}+\frac{1}{2} \mu_{22}-\frac{1}{2} \mu_{33}\right)\right]$.

There are two ways to satisfy (5.1) without any unnatural cancellation, at least one of which is obligatory. Either we must have option (1), namely that $m_{\nu_{2}}^{0}=m_{\nu_{1}}^{0}$ and $\mu_{22}=\mu_{33}$ or there must be option (2), namely that $m_{\nu_{3}}^{0}, m_{\nu_{2}}^{0}, m_{\nu_{1}}^{0}, \mu_{12}+\mu_{13}$ and $\mu_{22}-\mu_{33}$ are all real so that each side of (5.1) vanishes. Take (1) first. Since $m_{\nu 1}^{0}=\left|m_{\nu 1}^{0}\right|$ by choice, we now have $\left|m_{\nu_{1}}^{0}\right|=\left|m_{\nu_{2}}^{0}\right|$ and $\alpha_{21}^{0}=0$, i.e. $\Delta_{21} \equiv\left|m_{\nu 2}^{2}\right|-\left|m_{\nu 1}\right|^{2}$ arises solely from TBM breaking. Further, with $\mu_{22}=\mu_{33}$, the implication from from (4.24) and (4.25) is that $\sqrt{2} \operatorname{Re} \epsilon_{23}^{\nu}=$ $\operatorname{Re} \epsilon_{13}^{\nu}+O\left(\epsilon^{2}\right)$. Consequently, it follows from (4.3) and (4.6) that $c_{23}-s_{23}=-\sqrt{2} \epsilon_{23}^{\ell}+O\left(\epsilon^{2}\right)$ which leads to the result $\left|s_{23}-\frac{1}{\sqrt{2}}\right|=\frac{1}{\sqrt{2}}\left|\epsilon_{23}^{\ell}\right|+\left|O\left(\epsilon^{2}\right)\right| \ll\left|O\left(\epsilon^{\nu}\right)\right|$. The strong inequality in the last step has been based on the discussion which followed (4.14). Thus option (1) says that the magnitude of any deviation from maximal atmospheric mixing, being of order $\left|\epsilon_{23}^{\ell}\right|$ and small, will not be easily observed in forthcoming experiments. Let us turn to alternative (2). Now we have $\alpha_{21}^{0}$ and $\alpha_{31}^{0}$ equalling 0 or $\pi$. Further, by use of (4.19) and (4.24), we derive that $\operatorname{Im} \epsilon_{23}^{\nu}=O\left(\epsilon^{2}\right)$. As a result, by virtue of (4.16) as well as (4.17), one concludes that $s_{13} \sin \delta_{\mathrm{CP}}=O\left(\epsilon^{2}\right)$ and $J=O\left(\epsilon^{2}\right)$, so that both would be small and hard to detect in experiments planned for the near future. The implication of option (2) is that $\mathrm{CP}$ violation in neutrino oscillations may not be seen in those experiments. It may be noted that the assumption $\left|\epsilon^{\ell}\right| \ll\left|\epsilon^{\nu}\right|$ is unnecessary for this option.

It is also noteworthy that in option (1) one needs to use degenerate perturbation theory [31-33] with respect to the TBM limit for the 1-2 sector of neutrinos. In the latter case, the perturbation splits the 1-2 mass degeneracy and generates the solar neutrino mass difference with $m_{\nu 1}^{0}=m_{\nu 2}^{0}=m_{\nu}^{0}$. One then obtains

$$
\Delta_{21}=\sqrt{\left(p_{11}^{\nu}-p_{22}^{\nu}\right)^{2}+p_{12}^{\nu}}
$$

as calculated using (3.14). Additionally, to order $\epsilon^{\nu}$ and $\epsilon^{\ell}, s_{13} e^{i \delta_{\mathrm{CP}}}$ can be obtained in terms of $m_{\nu 3}^{0}, m_{\nu}^{0}, m_{e}^{0}, m_{\mu}^{0}, m_{\tau}^{0}$ as well as the $\mu$ and $\lambda$ parameters by using (4.7) and employing the expressions for the $\epsilon$ parameters. We choose not write that full expression here. 
Some comments on the issue of unnatural cancellations are in order. The TBM breaking terms in the mass matrix of charged leptons do not leave any residual symmetry except possibly some rephasing invariances. As stated earlier, given that $m_{e} \ll m_{\mu} \ll m_{\tau}$, the cancellations required to avoid the reality condition on all $\lambda_{i j}$ (for $i \neq j$ ) cannot be effected by any such invariance. In the neutrino case, there generally is a residual $Z_{2}$ symmetry [711, 14-16] after TBM is broken. Even such a discrete symmetry does not generally enable one to obtain the concerned complicated equality between specific combinations of TBM violating perturbation parameters, TBM invariant neutrino masses as well as Majorana phases. We feel, therefore, that our argument ruling out such cancellations is sound and our conclusions are reliable.

Let us finally remark on the relevance of our result to planned experiments at the proton beam intensity frontier. The determination of the sign of the neutrino mass ordering is one of their aims. It is noteworthy that the constraint on neutrino mixing parameters, derived by us, is independent of this issue just as the consequences of exact TBM are. Those experiments will also investigate neutrino mixing parameters. A combination [34-36] of data from the ongoing and upcoming runs of $\mathrm{T} 2 \mathrm{~K}$ and $\mathrm{NO} \nu \mathrm{A}$ experiments would probe $\left|s_{23}-\frac{1}{\sqrt{2}}\right|$ from the conversion probability $P\left(\nu_{\mu} \rightarrow \nu_{e}\right)$. Now, in case a sizable nonzero value of that quantity is measured, being of magnitude comparable in percentage terms to (100 $\left.s_{13}\right) \%$ of the maximal value of $s_{23}$, our condition (2) would hold and predict a small amount of $\mathrm{CP}$ violation in neutrino oscillations from the above data. Contrariwise, the failure to measure any deviation from maximal atmospheric neutrino mixing outside error bars would mean that our condition (1) would operate with $s_{13} \sin \delta_{\mathrm{CP}}=O\left(\epsilon^{\nu}\right), J=O\left(\epsilon^{\nu}\right)$ permitted; that would bolster the hope of detecting CP nonconservation for oscillating neutrinos from the difference in conversion probabilities $P\left(\nu_{\mu} \rightarrow \nu_{e}\right)-P\left(\overline{\nu_{\mu}} \rightarrow \overline{\nu_{e}}\right)$. The latter would be good news not only for a combined analysis of data from forthcoming runs of [37] of $\mathrm{T} 2 \mathrm{~K}$ and $\mathrm{NO} \nu \mathrm{A}$ but also for future experiments with superbeams, such as LBNF [38], LBNO [39, 40] or a neutrino factory at $10 \mathrm{GeV}$ [41]. Current hints, either for a non-maximal $\theta_{23}$ or a nonzero $\sin \delta_{\mathrm{CP}} / J$, by no means constitute any robust evidence and an experimental resolution of these two issues is urgently called for.

\section{Comparative studies with specific flavor models of broken TBM}

In the present analysis we have used first order perturbation theory to analytically establish relations between basis independent sets of small coefficients $\left\{\epsilon^{\ell}, \epsilon^{\nu}\right\}$ and TBM deviant measurables. In doing so, we have been able to establish the relations given in eq. (4.1) to eq. (4.4). Physical observables partaining to CP violation have also been related analytically to these basis independent $\epsilon$ coefficients. For these relations to remain valid, TBM symmetry should be broken weakly so that one could jusify first order perturbation theory. If that symmetry is broken strongly, in other words, if the Lagrangian contains large terms violating TBM symmetry, then these relations would fail to be true. ${ }^{4}$ In that case direct numerical diagonalization would need to be made. On the other hand, numerical

\footnotetext{
${ }^{4}$ TBM braking in general is naturally expected to be under control for lowest order perturbation theory since $s_{13}$ has been observed to be $<0.18$.
} 
diagonalization cannot be done in a model independent way; consequently, a case by case study, depending on the model of TBM symmetry breaking, would be required.

Given our two assumptions, namely (1) $\left|\epsilon^{\ell}\right| \ll\left|\epsilon^{\nu}\right|$ and (2) the absence of unnatural cancellations, it is desirable to cross check our result with specific flavor symmetry models which break tribimaximality by some amount. We consider below several such proposed models in a representative but not comprehensive survey. Most (though not all) of these are variations of a basic family symmetry model [42] utilizing the discrete group $A_{4}$ along with gauge singlet Higgs fields called flavons which transform as specified $A_{4}$ representations. Not every such model can be cast within the framework of additive perturbations to $M_{\nu f}^{0}$ and $M_{\ell f}^{0}$. Nonetheless, we deem it useful to make this comparison. In these models, if some flavons develop VEVs aligned in appropriately chosen directions in the corresponding $A_{4}$ representation space, TBM obtains in the neutrino sector with mass diagonal charged leptons. Certain higher mass dimensional terms are entered into the Lagrangian containing ratios of flavon VEVs divided by a much larger cut-off scale. If some slight misalignment is then introduced in these VEV directions, deviations result from exact TBM.

The first example of this type on our list is that of ref. [43] which utilizes two $A_{4}$ triplet and three $A_{4}$ singlet flavons. An analytical study of this model was made here while the TBM deviants were investigated numerically. The magnitude of the dominant TBM breaking parameters was restricted to small values by taking $\left|U_{13}\right|<0.2$. This analysis took care to ensure unitary implementation of broken TBM symmetry, i.e. that perturbed eigenstates of type $t$ do make up the columns of $U_{t}$. A revealing facet of this model is that the misalignment induced coefficients in the perturbed charged lepton eigenstates turn out to be significantly less in magnitude than the corresponding ones for neutrinos. This is since the latter get enhanced by mass ratio factors such as $\left(m_{\nu 1}^{0}+m_{\nu 3}^{0}\right)\left(m_{\nu 2}^{0}-\right.$ $\left.m_{\nu 1}^{0}\right)^{-1},\left(m_{\nu 1}^{0}+m_{\nu 3}^{0}\right)\left|m_{\nu 2}^{0}-m_{\nu 3}^{0}\right|^{-1}$ and $\left(m_{\nu 3}^{0}+m_{\nu 1}^{0}\right)\left|m_{\nu 1}^{0}-m_{\nu 3}^{0}\right|^{-1}$ in our notation, from the imposed unitary implementation. The corresponding factors in the charged lepton case are non-enhancing because of the hierarchical nature of the charged lepton masses. Thus the model manifestly satisfies our condition $\left|\epsilon^{\ell}\right|<\left|\epsilon^{\nu}\right|$. The computed numerical values of $J$ are found to go all the way up to 0.046 when the full parameter space is scanned, cf. table II of ref. [43]. This means that $\left(s_{13} \sin \delta_{\mathrm{CP}}\right)_{\max } \sim 0.195$, allowing substantial possible $\mathrm{CP}$ violation in neutrino oscillations. However, throughout the parameter space, one always has $\sin ^{2} 2 \theta_{23}<0.994$ i.e. $\left|s_{23}-\sqrt{\frac{1}{2}}\right|<0.03$, which permits only a tiny deviation from maximality in atmospheric atmospheric neutrino mixig. Therefore this model satisfies our option (1). The second model [44] that we consider is very similar to that of ref. [43] except that the perturbations can be arbitrarily large and real flavon VEVs were chosen; consequently, there is no $\mathrm{CP}$ violation to be observed in neutrino oscillations. The deviation from maximality in $s_{23}$ can be made large only by chosing the TBM breaking perturbation parameter $\left|U_{e 3}\right| \sim 0.4$. If $\left|U_{e 3}\right|$ is restricted to $<0.2$, as dictated by later experiments, once again the numerical constraint $\left|s_{23}-\sqrt{\frac{1}{2}}\right|<0.03$ is seen to operate in agreement with ref. [43], i.e. the deviation from maximal neutrino mixing is small by our critarion. Hence our option (1) is maintained here with the additional proviso of a nonexistent $J$. Significant deviations $\left|s_{23}-\sqrt{\frac{2}{3}}\right|$ can occur for very large perturbations which are beyond the scope of our work. 
We then consider the study of $A_{4}$ and $S_{4}$ based flavor symmetry models with perturbed lepton mass matrices reported in ref. [45]. In particular, for the $S_{4}$ based model investigated, $s_{23}^{2}$ gets fixed at $1 / 2$ and there is no deviation from maximality in atmospheric neutrino mixing; moreover, $\delta_{\mathrm{CP}}$ is preferred to be near $\pi$, i.e. no significant CP-violation in neutrino oscillations is predicted. So this model is compatible with both our options 1 and 2. In the $A_{4}$ based model considered (with just two $A_{4}$ singlet flavons), the authors derive the sum rule $s_{13}^{2} \sin ^{2} \delta_{\mathrm{CP}}=s_{13}^{2}-2\left(s_{23}^{2}-1 / 2\right)^{2}$. It is noteworthy that both our options 1 and 2 are compatible with this result. This is since, according to the sum rule, $\mathrm{CP}$ violation in neutrino oscillations is largest when $s_{23}^{2}=1 / 2$ while the deviation from maximal atmospheric neutrino mixing is greatest when the Dirac phase $\delta_{\mathrm{CP}}=0$ or $\pi$ i.e. there is no $\mathrm{CP}$ violation.

The next analysis in our menu is that of ref. [46]. Here again $A_{4}$-based models are considered with the number of $A_{4}$ singlet flavons varying from one to three and with the possibility of including the see-saw mechanism for neutrino mass generation. Additive perturbations are considered vis-à-vis TBM invariant charged lepton and neutrino mass matrices and numerical diagonalization is carried out. The parameter spaces of the models considered here allow both a substantial $J \sim 0.02$ (i.e. $\delta_{\mathrm{CP}} \sim 30^{\circ}$ ) and a sizable $\left|s_{23}^{2}-1 / 2\right| \geq$ 0 . However, unlike in ref. [43], very large perturbation parameters have been alowed here. For instance, the charged lepton perturbations $\epsilon^{\text {ch }}$ have been taken upto 0.3 while the corresponding neutrino ones have been kept completely free in the numerical scan with large allowed values. Thus lowest order perturbation theory does not apply to a considerable region of their parameter space. We expect that their results should agree with those of ref. [43] once the smallness criterion is imposed on the perturbations.

The final analysis within the ambit of our comparative study is that of ref. [47]. This work is somewhat different from the previously considered models in that no specific flavor symmetry such as $A_{4}$ for the Lagrangian is assumed. Instead, three separate mechanisms of TBM breaking are considered per se: (1) corrections to $U_{\ell}$ in the charged lepton sector while keeping $U_{\nu}^{0}$ unchanged, (2) renormalization group corrections (with supersymmetry) starting from exact TBM and nearly mass degenerate neutrinos at a very high scale and (3) explicit TBM breaking terms added to $M_{\nu f}^{0}$ in the neutrino sector only. For (1), the authors find that $J$ approaches a near maximum with $\delta_{\mathrm{CP}} \sim \pi / 2$ but the deviation from maximal atmospheric neutrino mixing is small with $s_{23}^{2}=1 / 2+O\left(\left|U_{e 3}\right|^{2}\right)$. This respects our option (1). For cases (2) and (3) of ref. [47], sizable such deviations in the latter are possible with $\left|s_{23}^{2}-1 / 2\right| \sim 0.1-0.2$; however, $J$ was not investigated. For case (2), in particular, exact TBM at a high scale makes the starting boundary value of $\delta_{\mathrm{CP}}$ indeterminate and an unambiguous answer is not possible.

\section{Concluding summary}

In this paper we have considered general perturbations at the lowest order to hermitian squared mass matrices $M_{\ell f}^{\dagger} M_{\ell f}$ and $M_{\nu f}^{\dagger} M_{\nu f}$ respectively for charged leptons and neutrinos in the flavor basis of each and away from their TBM limits by carefully taking into account the unitary relation between the mass basis and the flavor basis. We have utilized the fact 
that columns of the said unitary matrix are the perturbed eigenstates. We have derived linear expressions for the three measurable TBM deviants in terms of the dimensionless coefficients that appear in the perturbed charged lepton and neutrino eigenstates. We have further derived four independent constraints on the imaginary parts of the latter from the requirement that four of the elements of $U_{\ell}^{\dagger} U_{\nu}$ have to be real. With the plausible arguements of the mixing caused by the strongly mass hierarchical charged leptons being significantly smaller than that due to neutrinos and no unnatural cancellations, we have derived a result, forcing one of two possibilities, which should be testable in the foreseeable future. This main result of ours can be stated succintly in the language of mathematical logic. Proposition A: an accurate description of neutrino mixing is given by the lowest order of additively perturbed tribimaximality without unnatural cancellations and with the mixing from the strongly mass hierarchical charged leptons being significantly smaller than that from neutrinos. Proposition B: $\left|s_{23}-\sqrt{\frac{1}{2}}\right|=O\left(\epsilon^{\nu}\right)$. Proposition C: $s_{13} \sin \delta_{\mathrm{CP}} / J=$ $O\left(\epsilon^{\nu}\right)$. Then $A \cap(B \cup C)=\emptyset$.

\section{A Derivation of mixing constraints}

Neglecting $O\left(\epsilon^{2}\right)$ terms, we may write,

$$
\begin{aligned}
& \left|\psi_{1}^{\nu, l}\right\rangle_{f}=\left|\psi_{1}^{0 \nu, l}\right\rangle_{f}+\epsilon_{12}^{\nu, l}\left|\psi_{2}^{0 \nu, l}\right\rangle_{f}+\epsilon_{13}^{\nu, l}\left|\psi_{3}^{0 \nu, l}\right\rangle_{f}, \\
& \left|\psi_{2}^{\nu, l}\right\rangle_{f}=-\epsilon_{12}^{\nu, l^{*}}\left|\psi_{1}^{0 \nu, l}\right\rangle_{f}+\left|\psi_{2}^{0 \nu, l}\right\rangle_{f}+\epsilon_{23}^{\nu, l}\left|\psi_{3}^{0 \nu, l}\right\rangle_{f}, \\
& \left|\psi_{3}^{\nu, l}\right\rangle_{f}=-\epsilon_{12}^{\nu, l^{*}}\left|\psi_{1}^{0 \nu, l}\right\rangle_{f}-\epsilon_{23}^{\nu, l^{*}}\left|\psi_{2}^{0 \nu, l}\right\rangle_{f}+\left|\psi_{3}^{0 \nu, l}\right\rangle_{f},
\end{aligned}
$$

where

$$
\left|\psi_{1}^{0 \nu}\right\rangle_{f}=\left(\begin{array}{c}
\sqrt{\frac{2}{3}} \\
-\sqrt{\frac{1}{6}} \\
\sqrt{\frac{1}{6}}
\end{array}\right), \quad\left|\psi_{2}^{0 \nu}\right\rangle_{f}=\left(\begin{array}{c}
\sqrt{\frac{1}{3}} \\
\sqrt{\frac{1}{3}} \\
-\sqrt{\frac{1}{3}}
\end{array}\right), \quad\left|\psi_{3}^{0 \nu}\right\rangle_{f}=\left(\begin{array}{c}
0 \\
\sqrt{\frac{1}{2}} \\
\sqrt{\frac{1}{2}}
\end{array}\right)
$$

and

$$
\left|\psi_{1}^{0 l}\right\rangle_{f}=\left(\begin{array}{l}
1 \\
0 \\
0
\end{array}\right), \quad\left|\psi_{2}^{0 l}\right\rangle_{f}=\left(\begin{array}{l}
0 \\
1 \\
0
\end{array}\right), \quad\left|\psi_{3}^{0 l}\right\rangle_{f}=\left(\begin{array}{l}
0 \\
0 \\
1
\end{array}\right) .
$$

By using $U_{\ell}=\left(\left|\psi_{1}^{\ell}\right\rangle_{f}\left|\psi_{2}^{\ell}\right\rangle_{f}\left|\psi_{2}^{\ell}\right\rangle_{f}\right)$ and $U_{\nu}=\left(\left|\psi_{1}^{\nu}\right\rangle_{f}\left|\psi_{2}^{\nu}\right\rangle_{f}\left|\psi_{3}^{\nu}\right\rangle_{f}\right)$, one is led to the respective expressions for $U_{\ell}$ and $U_{\nu}$, as given in the text. If we define $L \equiv U_{\ell}^{\dagger} U_{\nu}$, then neglecting $O\left(\epsilon^{2}\right)$ terms, the nine elements of the $L$ matrix are

$$
\begin{aligned}
& L_{11}=\sqrt{\frac{2}{3}}+\sqrt{\frac{1}{3}} \epsilon_{12}^{\nu}-\sqrt{\frac{1}{6}} \epsilon_{12}^{\ell *}+\sqrt{\frac{1}{6}} \epsilon_{13}^{\ell *}, \\
& L_{12}=\sqrt{\frac{1}{3}}-\sqrt{\frac{2}{3}} \epsilon_{12}^{\nu *}+\sqrt{\frac{1}{3}} \epsilon_{12}^{\ell *}-\sqrt{\frac{1}{3}} \epsilon_{13}^{\ell *}, \\
& L_{13}=-\sqrt{\frac{2}{3}} \epsilon_{13}^{\nu *}-\sqrt{\frac{1}{3}} \epsilon_{23}^{\nu *}+\sqrt{\frac{1}{2}} \epsilon_{12}^{\ell *}+\sqrt{\frac{1}{2}} \epsilon_{13}^{\ell *},
\end{aligned}
$$




$$
\begin{aligned}
& L_{21}=-\sqrt{\frac{1}{6}}+\sqrt{\frac{1}{3}} \epsilon_{12}^{\nu}+\sqrt{\frac{1}{2}} \epsilon_{13}^{\nu}-\sqrt{\frac{2}{3}} \epsilon_{12}^{\ell}+\sqrt{\frac{1}{6}} \epsilon_{23}^{\ell *}, \\
& L_{22}=\sqrt{\frac{1}{3}}+\sqrt{\frac{1}{6}} \epsilon_{12}^{\nu *}+\sqrt{\frac{1}{2}} \epsilon_{23}^{\nu}-\sqrt{\frac{1}{3}} \epsilon_{12}^{\ell}-\sqrt{\frac{1}{3}} \epsilon_{12}^{\ell *}, \\
& L_{23}=\sqrt{\frac{1}{2}}+\sqrt{\frac{1}{6}} \epsilon_{13}^{\nu *}-\sqrt{\frac{1}{3}} \epsilon_{23}^{\nu *}+\sqrt{\frac{1}{2}} \epsilon_{23}^{\ell *}, \\
& L_{31}=\sqrt{\frac{1}{6}}-\sqrt{\frac{1}{3}} \epsilon_{12}^{\nu}+\sqrt{\frac{1}{2}} \epsilon_{13}^{\nu}-\sqrt{\frac{2}{3}} \epsilon_{13}^{\ell}+\sqrt{\frac{1}{6}} \epsilon_{23}^{\ell}, \\
& L_{32}=-\sqrt{\frac{1}{3}}-\sqrt{\frac{1}{6}} \epsilon_{12}^{\nu *}+\sqrt{\frac{1}{2}} \epsilon_{23}^{\nu}-\sqrt{\frac{1}{3}} \epsilon_{13}^{\ell}-\sqrt{\frac{1}{3}} \epsilon_{23}^{\ell}, \\
& L_{33}=\sqrt{\frac{1}{2}}-\sqrt{\frac{1}{6}} \epsilon_{13}^{\nu *}+\sqrt{\frac{1}{3}} \epsilon_{23}^{\nu *}-\sqrt{\frac{1}{2}} \epsilon_{23}^{\ell} .
\end{aligned}
$$

Similarly, defining $N \equiv U_{P M N S} K^{-1}$ and again neglecting $O\left(\epsilon^{2}\right)$ terms

$$
N=\left(\begin{array}{ccc}
c_{12} & s_{12} & s_{13} e^{-i \delta_{\mathrm{CP}}} \\
-s_{12} c_{23}-\sqrt{\frac{1}{3}} s_{13} e^{i \delta_{\mathrm{CP}}} & c_{12} c_{23}-\sqrt{\frac{1}{6}} s_{13} e^{i \delta_{\mathrm{CP}}} & s_{23} \\
s_{12} s_{23}-\sqrt{\frac{1}{3}} s_{13} e^{i \delta_{\mathrm{CP}}} & -c_{12} s_{23}-\sqrt{\frac{1}{6}} s_{13} e^{i \delta_{\mathrm{CP}}} & c_{23}
\end{array}\right) .
$$

Expanding in $\epsilon$, the relations $\sqrt{2} c_{12}+s_{12}=\sqrt{3}+O\left(\epsilon^{2}\right)$ and $c_{23}+s_{23}=\sqrt{2}+O\left(\epsilon^{2}\right)$ are automatic. The equality $L=N$ leads to the mixing constraint relations. Specifically, the identification of elements or their combinations

$$
\begin{array}{lll}
L_{11}=N_{11}, & L_{21}-L_{31}=N_{21}-N_{31}, & L_{21}+L_{31}=N_{21}+N_{31}, \\
L_{12}=N_{12}, & L_{22}+L_{32}=N_{22}+N_{32}, & L_{22}-L_{32}=N_{22}-N_{32}, \\
L_{13}^{*}=N_{13}^{*}, & L_{33}+L_{23}=N_{33}+N_{23}, & L_{33}-L_{23}=N_{33}-N_{23},
\end{array}
$$

neglecting $O\left(\epsilon^{2}\right)$ terms, lead respectively to the equations

$$
\begin{aligned}
& c_{12}-\sqrt{\frac{2}{3}}=\frac{1}{\sqrt{2}}\left(\sqrt{\frac{1}{3}}-s_{12}\right)=\sqrt{\frac{1}{3}} \epsilon_{12}^{\nu}-\sqrt{\frac{1}{6}}\left(\epsilon_{12}^{\ell *}-\epsilon_{13}^{\ell *}\right), \\
& -\sqrt{\frac{1}{3}}\left(c_{23}-s_{23}\right)-\frac{2}{\sqrt{3}} s_{13} e^{i \delta_{\mathrm{CP}}}=\sqrt{2} \epsilon_{13}^{\nu}-\sqrt{\frac{2}{3}}\left(\epsilon_{12}^{\ell}+\epsilon_{13}^{\ell}\right)+\sqrt{\frac{1}{6}}\left(\epsilon_{23}^{\ell}+\epsilon_{23}^{\ell *}\right), \\
& -\sqrt{2} s_{12}=-2 \sqrt{\frac{1}{6}}+2 \sqrt{\frac{1}{3}} \epsilon_{12}^{\nu}-\sqrt{\frac{2}{3}}\left(\epsilon_{12}^{\ell}-\epsilon_{13}^{\ell}\right)+\sqrt{\frac{1}{6}}\left(\epsilon_{23}^{\ell}-\epsilon_{23}^{\ell *}\right), \\
& s_{12}=\sqrt{\frac{1}{3}}-\sqrt{\frac{2}{3}} \epsilon_{12}^{\nu *}+\sqrt{\frac{1}{3}}\left(\epsilon_{12}^{\ell *}-\epsilon_{13}^{\ell *}\right), \\
& \sqrt{\frac{2}{3}}\left(c_{23}-s_{23}-s_{13} e^{i \delta_{\mathrm{CP}}}\right)=\sqrt{2} \epsilon_{23}^{\nu}-\sqrt{\frac{1}{3}}\left(\epsilon_{12}^{\ell}+\epsilon_{13}^{\ell}+\epsilon_{23}^{\ell *}+\epsilon_{23}^{\ell}\right), \\
& \sqrt{2} c_{12}=2 \sqrt{\frac{1}{3}}+2 \sqrt{\frac{1}{6}} \epsilon_{12}^{\nu *}-\sqrt{\frac{1}{3}}\left(\epsilon_{23}^{\ell *}-\epsilon_{23}^{\ell}\right)-\sqrt{\frac{1}{3}}\left(\epsilon_{12}^{\ell}-\epsilon_{13}^{\ell}\right),
\end{aligned}
$$




$$
\begin{aligned}
s_{13} e^{i \delta_{\mathrm{CP}}} & =-\sqrt{\frac{1}{3}}\left(\sqrt{2} \epsilon_{13}^{\nu}+\epsilon_{23}^{\nu}\right)+\sqrt{\frac{1}{2}}\left(\epsilon_{12}^{\ell}+\epsilon_{13}^{\ell}\right) \\
c_{23}+s_{23} & =\sqrt{2}+\frac{1}{\sqrt{2}}\left(\epsilon_{23}^{\ell *}-\epsilon_{23}^{\ell}\right), \\
c_{23}-s_{23} & =-\sqrt{\frac{2}{3}} \epsilon_{13}^{\nu *}-\sqrt{\frac{1}{2}}\left(\epsilon_{23}^{\ell}+\epsilon_{23}^{\ell *}\right)+\frac{2}{\sqrt{3}} \epsilon_{23}^{\nu *} .
\end{aligned}
$$

Eq. (4.3) is a direct conseqence of (A.23). Eq. (4.2) is easily derived from (A.20) and (A.22), while eq. (4.1) follows from (A.19) and (A.21). Now eq. (4.4) obtains from (A.21), whereas eq. (4.5) is just a rewritten form of (A.16) with the input of eq. (4.4). Eq. (4.6) follows from (A.17) and (A.20). Finally, eq. (4.7) is the same as (A.22).

\section{Acknowledgments}

A part of this work was done at the WHEPP13 workshop, Puri, India. We thank A. Dighe, P. Byakti, S. Choubey, A. Raychaudhuri and S. Uma Sankar for their comments. PR acknowledges a Senior Scientistship of Indian National Science Academy.

Open Access. This article is distributed under the terms of the Creative Commons Attribution License (CC-BY 4.0), which permits any use, distribution and reproduction in any medium, provided the original author(s) and source are credited.

\section{References}

[1] Particle Data Group collaboration, J. Beringer et al., Review of Particle Physics (RPP), Phys. Rev. D 86 (2012) 010001 [inSPIRE].

[2] R.N. Mohapatra and A.Y. Smirnov, Neutrino Mass and New Physics, Ann. Rev. Nucl. Part. Sci. 56 (2006) 569 [hep-ph/0603118] [InSPIRE].

[3] Intensity Frontier Neutrino Working Group collaboration, A. de Gouvêa et al., Working Group Report: Neutrinos, arXiv:1310.4340 [INSPIRE].

[4] S. Parke, Neutrinos: Theory and Phenomenology, Phys. Scripta T 158 (2013) 014013 [arXiv: 1310.5992] [INSPIRE].

[5] H. Minakata, Neutrino Physics Now and in the Near Future, arXiv:1403.3276 [INSPIRE].

[6] J. Lesgourgues and S. Pastor, Neutrino mass from Cosmology, Adv. High Energy Phys. 2012 (2012) 608515 [arXiv: 1212.6154] [INSPIRE].

[7] G. Atlarelli and F. Feruglio, Models of neutrino masses and mixings, New J. Phys. 6 (2006) 106 [hep-ph/0405048] [INSPIRE].

[8] G. Altarelli anc F. Feruglio, Theoretical models of neutrino masses and mixings, Springer Tracts Mod. Phys. 190 (2003) 169 [hep-ph/0206077] [INSPIRE].

[9] S. King and C. Luhn, Neutrino mass and mixing with discrete symmetry, Rept. Prog. Phys. 76 (2013) 056201 [arXiv:1301.1340] [InSPIRE].

[10] P.F. Harrison, D.H. Perkins and W.G. Scott, Tri-bimaximal mixing and the neutrino oscillation data, Phys. Lett. B 530 (2002) 167 [hep-ph/0202074] [INSPIRE]. 
[11] G. Altarelli, F. Feruglio and L. Merlo, Tri-Bimaximal Neutrino Mixing and Discrete Flavour Symmetries, Fortsch. Phys. 61 (2013) 507 [arXiv:1205.5133] [INSPIRE].

[12] S.-F. Ge, D.A. Dicus and W.W. Repko, $Z_{2}$ Symmetry Prediction for the Leptonic Dirac CP Phase, Phys. Lett. B 702 (2011) 220 [arXiv:1104.0602] [INSPIRE].

[13] S.F. King and C. Luhn, Trimaximal neutrino mixing from vacuum alignment in A4 and S4 models, JHEP 09 (2011) 042 [arXiv: 1107.5332] [INSPIRE].

[14] S.-F. Ge, D.A. Dicus and W.W. Repko, Residual Symmetries for Neutrino Mixing with a Large $\theta_{13}$ and Nearly Maximal $\delta_{D}$, Phys. Rev. Lett. 108 (2012) 041801 [arXiv:1108.0964] [INSPIRE].

[15] D. Hernandez and A.Y. Smirnov, Lepton mixing and discrete symmetries, Phys. Rev. D 86 (2012) 053014 [arXiv: 1204.0445] [InSPIRE].

[16] A.D. Hanlon, W.W. Repko and D.A. Dicus, Residual Symmetries Applied to Neutrino Oscillations at NOvA and T2K, Adv. High Energy Phys. 2014 (2014) 469572 [arXiv: 1403.7552] [INSPIRE].

[17] G. Altarelli, S. Feruglio, L. Merlo and E. Stamou, Discrete symmetry groups, $\theta_{13}$ and lepton flavor violation, JHEP 08 (2012) 021 [arXiv:1205.4670] [INSPIRE].

[18] A.D. Hanlon, S.-F. Ge and W.W. Repko, Phenomenological consequences of residual $\mathbb{Z}_{2}^{s}$ and $\overline{\mathbb{Z}}_{2}^{s}$ symmetries, Phys. Lett. B 729 (2014) 185 [arXiv: 1308.6522] [INSPIRE].

[19] M.C. Gonzalez-Garcia, M. Maltoni, J. Salvado and T. Schwetz, Global fit to three neutrino mixing: critical look at present precision, JHEP 12 (2012) 123 [arXiv:1209.3023] [INSPIRE].

[20] D.V. Forero, M. Tortola and J.W.F. Valle, Global status of neutrino oscillation parameters after Neutrino-2012, Phys. Rev. D 86 (2012) 073012 [arXiv:1205.4018] [INSPIRE].

[21] F. Capozzi, G.L. Fogli, E. Lisi, A. Marrone, D. Montanino and A. Palazzo, Status of three-neutrino oscillation parameters, circa 2013, Phys. Rev. D 89 (2014) 093018 [arXiv:1312.2878] [INSPIRE].

[22] DAYA-BAY collaboration, F.P. An et al., Observation of electron-antineutrino disappearance at Daya Bay, Phys. Rev. Lett. 108 (2012) 171803 [arXiv:1203.1669] [INSPIRE].

[23] RENO collaboration, J.K. Ahn et al., Observation of Reactor Electron Antineutrino Disappearance in the RENO Experiment, Phys. Rev. Lett. 108 (2012) 191802 [arXiv: 1204.0626] [INSPIRE].

[24] MINOS collaboration, P. Adamson et al., Improved search for muon-neutrino to electron-neutrino oscillations in MINOS, Phys. Rev. Lett. 107 (2011) 181802 [arXiv: 1108.0015] [INSPIRE].

[25] DOUBLE-CHOOZ collaboration, Y. Abe et al., Indication for the disappearance of reactor electron antineutrinos in the Double CHOOZ experiment, Phys. Rev. Lett. 108 (2012) 131801 [arXiv:1112.6353] [INSPIRE].

[26] Z.-z. Xing, Nearly tri bimaximal neutrino mixing and CP-violation, Phys. Lett. B 533 (2002) 85 [hep-ph/0204049] [INSPIRE].

[27] X.-G. He and A. Zee, Minimal Modification to Tri-bimaximal Mixing, Phys. Rev. D 84 (2011) 053004 [arXiv:1106.4359] [INSPIRE].

[28] D. Aristizabal Sierra, I. de Medeiros Varzielas and E. Houet, Eigenvector-based approach to neutrino mixing, Phys. Rev. D 87 (2013) 093009 [arXiv:1302.6499] [INSPIRE]. 
[29] D. Borah, Deviations from Tri-Bimaximal Neutrino Mixing Using Type II Seesaw, Nucl. Phys. B 876 (2013) 575 [arXiv:1307.2426] [INSPIRE].

[30] C. Soumya, K.N. Deepthi and R. Mohanta, A comprehensive study of the discovery potential

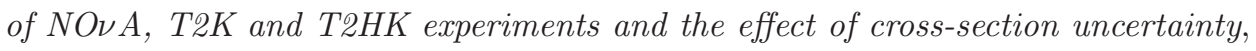
arXiv: 1408.6071 [INSPIRE].

[31] B. Brahmachari and A. Raychaudhuri, Perturbative generation of $\theta_{13}$ from tribimaximal neutrino mixing, Phys. Rev. D 86 (2012) 051302(R) [arXiv:1204.5619] [INSPIRE].

[32] L.J. Hall and G.G. Ross, Discrete Symmetries and Neutrino Mass Perturbations for $\theta_{13}$, JHEP 11 (2013) 091 [arXiv: 1303.6962] [INSPIRE].

[33] S. Pramanick and A. Raychaudhuri, Smallness of $\theta_{13}$ and the size of the solar mass splitting: Are they related?, Phys. Rev. D 88 (2013) 093009 [arXiv:1308.1445] [InSPIRE].

[34] S.K. Agarwalla, S. Prakash and S.U. Sankar, Resolving the octant of $\theta_{23}$ with T2K and

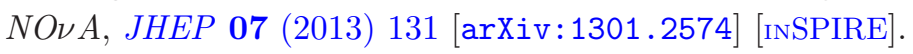

[35] A. Chatterjee, P. Ghoshal, S. Goswami and S.K. Raut, Octant sensitivity for large $\theta_{13}$ in atmospheric and long baseline neutrino experiments, JHEP 06 (2013) 010 [arXiv: 1302.1370] [INSPIRE].

[36] M. Ghosh, P. Ghoshal, S. Goswami and S.K. Raut, Evidence for leptonic CP phase from

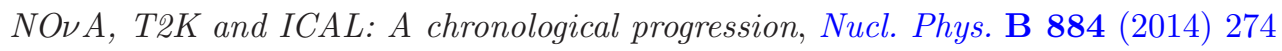
[arXiv: 1401.7243] [INSPIRE].

[37] P.A.N. Machado, H. Minakata, H. Nunokawa and R. Zukanovich Funchal, What can we learn about the lepton CP phase in the next 10 years?, JHEP 05 (2014) 109 [arXiv:1307.3248] [INSPIRE].

[38] LBNE collaboration, C. Adams et al., The Long-Baseline Neutrino Experiment: Exploring Fundamental Symmetries of the Universe, arXiv:1307.7335 [INSPIRE].

[39] A. Stahl et al., Expression of Interest for a very long baseline neutrino oscillation experiment (LBNO), CERN-SPSC-2012-021 [INSPIRE].

[40] LAGUNA-LBNO collaboration, S.K. Agarwalla et al., The mass-hierarchy and $C P$-violation discovery reach of the LBNO long-baseline neutrino experiment, JHEP 05 (2014) 094 [arXiv: 1312.6520] [INSPIRE].

[41] International Design Study of the Neutrino Factory, https://www.ids-nf.org/wiki/FrontPage.

[42] G. Altarelli and F. Feruglio, Tri-bimaximal neutrino mixing from discrete symmetry in extra dimensions, Nucl. Phys. B 720 (2005) 64 [hep-ph/0504165] [INSPIRE].

[43] M. Honda and M. Tanimoto, Deviation from tri-bimaximal neutrino mixing in $A_{4}$ flavor symmetry, Prog. Theor. Phys. 119 (2008) 583 [arXiv:0801.0181] [INSPIRE].

[44] B. Brahmachari, S. Choubey and M. Mitra, The A4 flavor symmetry and neutrino phenomenology, Phys. Rev. D 77 (2008) 073008 [Erratum ibid. D 77 (2008) 119901] [arXiv: 0801.3554] [INSPIRE].

[45] G. Altarelli, F. Feruglio, L. Merlo and E. Stamou, Discrete Flavour Groups, $\theta_{13}$ and Lepton Flavour Violation, JHEP 08 (2012) 021 [arXiv:1205.4670] [INSPIRE].

[46] J. Barry and W. Rodejohann, Deviations from tribimaximal mixing due to the vacuum expectation value misalignment in $A_{4}$ models, Phys. Rev. D 81 (2010) 093002 [Erratum ibid. D 81 (2010) 119901] [arXiv: 1003.2385] [INSPIRE].

[47] S. Goswami, S.T. Petcov, S. Ray and W. Rodejohann, Large $\left|U_{e 3}\right|$ and Tri-bimaximal Mixing, Phys. Rev. D 80 (2009) 053013 [arXiv:0907.2869] [INSPIRE]. 